\title{
A Confidence-Based Update Rule for Self-updating Human Face Recognition Systems
}

\author{
Sri-Kaushik Pavani ${ }^{1,2}$, Federico M. Sukno ${ }^{1,2}$, Constantine Butakoff ${ }^{1,2}$, \\ Xavier Planes ${ }^{1,2}$, and Alejandro F. Frangi ${ }^{1,2}$ \\ ${ }^{1}$ Center for Computational Imaging \& Simulation Technologies in Biomedicine \\ (CISTIB), Information \& Communications Technologies Department, Universitat \\ Pompeu Fabra, Pg. Circumvallacio 8, Barcelona 08003, Spain \\ 2 Networking Biomedical Research Center on Bioengineering, Biomaterials and \\ Nanomedicine (CIBER-BBN), Barcelona, Spain
}

\begin{abstract}
The aim of this paper is to present an automatic update rule to make a face recognition system adapt itself to the continuously changing appearance of users. The main idea is that every time the system interacts with a user, it adapts itself to include his or her current appearance, and thus, it always stays up-to-date. We propose a novel quality measure, which is used to decide whether the information just learnt from a user can be used to aggregate to what the system already knows. In the absence of databases that suit our needs, we present a publicly available database with 14, 279 images of 35 users and 74 impostors acquired in a span of 5 months. Experiments on this database show that the proposed measure is adequate for a system to learn the current appearance of users in a non-supervised manner.
\end{abstract}

\section{Introduction}

In this paper, we describe an update rule to make a Face Recognition System (FRS) adapt itself to the continuously changing appearance of a human face. In previous research [10, it has been shown that there is an approximately linear decrease in the accuracy of a FRS with respect to the time elapsed between acquisition of training and testing images of a person. The decrease in the accuracy is the result of changes in facial appearance because of factors such as ageing, health, skin tanning, facial hair growth, scars, etc.

Adapting the FRS to the current appearance of users is vital because an unupdated FRS may have difficulties recognizing its rightful owner after a few years of use. A common way to maintain the FRS up-to-date is by learning the current appearance of a user whenever he/she interatcs with it. In this approach, when a person is identified by the FRS, the facial image goes through a selection process that decides whether the information learnt from that image is reliable or not. If the extracted information is deemed reliable, then the corresponding image is added to the training set of a particular user. Later, the FRS is re-trained with the recently updated training set, and thus, it remains up-to-date with the current appearance of the user.

M. Tistarelli and M.S. Nixon (Eds.): ICB 2009, LNCS 5558, pp. 151-160, 2009.

(C) Springer-Verlag Berlin Heidelberg 2009 
A major risk in automatically incorporating new training images is the potential for a gradual corruption of the training set. Since images are added in a non-supervised manner, images on which one of the system components (e.g., face detection or segmentation) failed could also be added. This leads to the corruption of the information that is used to recognize users. For example, the image shown in Fig. 1a, should not be added to the training set because the segmentation of the face into its constituent parts is inaccurate and therefore, the information learnt from this image will be incorrect.

In order to determine how accurately the facial texture that has been extracted from a test image represents the current appearance of a user, we propose a confidence measure called total confidence. If an image has high total confidence, it will be included in the training set, otherwise, it will be ignored. In our experiments, we used this measure to automatically update the FRS for a period of four months. We observed that the FRS maintained its accuracy after the updates and that the training set was not corrupted during the update process.

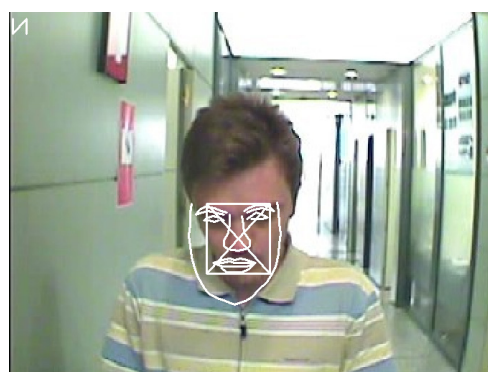

(a)

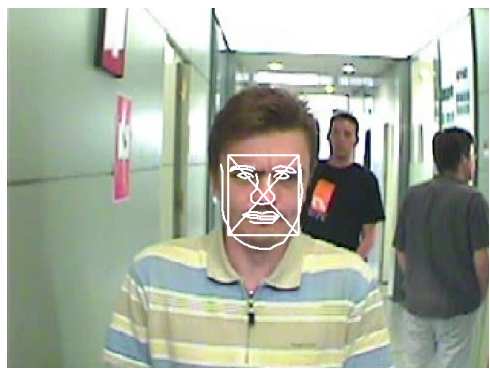

(b)

Fig. 1. Examples of bad (a) and good (b) facial segmentation. Including inaccurately segmented images, such as (a), corrupts the training set.

\section{Related Work}

Over the years, two main approaches were developed to handle the ever-changing appearance of human faces. In [3] and [4], the FRS is made invariant to ageing by simulating the effect of ageing on the appearance of a face. Such approaches are generally failure-prone, as the future appearance of a face is not only dependent on ageing, but also on other unpredictable factors such as permanent scarring due to accidents and effects of cosmetic surgery. Such an approach is primarily intended for applications where recent images of a person are not available. For example, they are used in predicting the current appearance of a person who has been missing for a long time.

There are applications where simulation of ageing becomes unnecessary. For example, a FRS installed at home interacts with the users almost on a daily basis. In such scenarios, the appearance of the users can be learnt every time the system sees a user, and therefore, the system can remain up-to-date. 
Aryananda [1] proposed and studied a humanoid robot (called Kismet) that has the ability to learn to recognize people during its interactions. The robot starts with an empty database and, as it interacts with people, it becomes a un-supervised FRS. In a reduced set of 9 individuals, the system was unable to learn 5 of them. It is not clear from their paper how the robot deals with cases when one of the elements of the recognition pipeline has been inaccurate.

Mou et al. 7] presented a technique to automatically create training databases for FRS in an unsupervised manner. The databases, once generated, can be used to automatically update a FRS. When a new face is input to the system, it is processed by the FaceVACS recognition technique which outputs the identity of a person and a similarity measure. If the similarity measure is greater than a preset threshold, the image is added to the training set. The authors produce results on 10 video sequences and they report that automatic construction of training sets occurred without corruption of data.

The main drawback of the above mentioned methods is that the results were reported on rather small databases, which questions the generalizability of their conclusions. One of the main reasons to use small databases is the unavailability of standard databases that have images of users captured on a daily basis over large periods of time. To this end, we present an image database containing 14, 279 images of 35 users and 74 impostors acquired on a day-to-day basis over five months. The database, called Gradual Evolution of Facial Appearance database (GEFA), can be obtained free of cost from http://cilab2.upf.edu/gefa/

\section{Face Recognition System}

A FRS was installed in a commonly visited area of our lab. The system, whose flowchart is shown in Fig. 2, has three main building blocks: face analysis, face identification and adaptive learning blocks.

\subsection{Face Analysis Block}

The face analysis block is responsible for detection and segmentation of human faces.

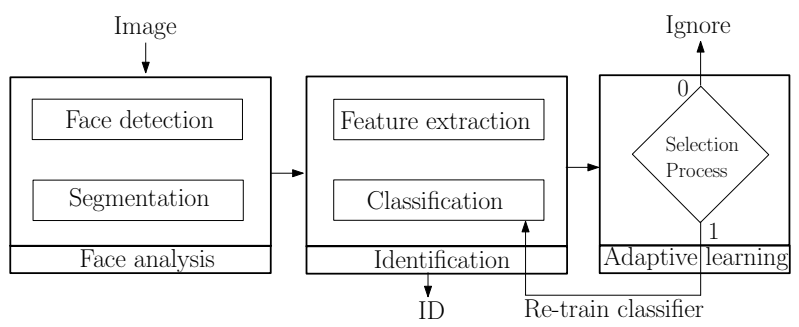

Fig. 2. Flow chart of the FRS. The input image is checked for the presence of faces. If any, the face is segmented and identified. In the selection process, the total confidence of the result is computed. This determines if the image is used to re-train the classifier. 
Face Detection. To detect faces accurately in real-time, our system employs Viola and Jones face detection procedure [13. The detector works at a speed of 15 frames per second at 12 resolution levels, while achieving low false acceptance rates.

Face Segmentation. For the segmentation of the prominent facial features our system employs Active Shape Models with Invariant Optimal Features (IOFASM) 12. This algorithm can automatically locate the outlines of eyes, mouth, nose, brows and silhouette contour of the face with an average error of approximately $2 \%$ of the inter-eye distance.

\subsection{Identification Block}

The identification block extracts texture from a segmented face and uses it to identify a user.

Facial Feature Extraction. To make facial texture independent of the facial expression, each image was warped using a piece-wise affine mapping between the result of the segmentation step and the mean shape. The texture parameters for each image were extracted by constructing an image intensity model of an Active Appearance Model [2].

Classification. Once the texture parameters are obtained, they are whitened [8] and then a distance-weighted $k$-Nearest Neighbor classifier $(k-\mathrm{NN})$ is used to identify the user in the image. The distance between two texture vectors is defined as the angle between them. The angle based distance metric was shown to be a good choice for PCA-based algorithms [9. All the experiments in this paper were performed using the five nearest neighbors $(k=5)$.

\subsection{Automatic Learning Block}

Every time a facial image has been identified, it goes through an automatic selection process that determines whether it is suitable to be added to the training set of the appropriate user. The selection process deems two categories of images to be unsuitable for automatic learning: 1) images with outdated facial appearance, 2 ) images where the algorithms used in our system produce unreliable results.

\section{Selection Process for Automatic Updates}

The selection process is based on the following four confidence measures:

1. Temporal confidence $\left(\mathcal{C}_{t}\right)$. The accuracy of FRSs has been shown to decrease linearly with the time elapsed between enrollment and testing [10. In our experiments, we assume that the maximum time limit of an image in representing a user's appearance is 5 years. The value of $\mathcal{C}_{t}$ is computed as in (11). 
The quantity $t_{e}$ stands for the time elapsed (in days) between the time of acquisition of the data and the current time.

$$
\mathcal{C}_{t}=\max \left(0,1-\frac{t_{e}}{1825}\right)
$$

2. Confidence of the face detector $\left(\mathcal{C}_{d}\right)$. The quantity $\mathcal{C}_{d}$ is computed in two steps. Firstly, the likelihood $p$ of a face is computed as the weighted ratio of the number of weak classifiers that label it as face to the total sum of weights of all the weak classifiers in the face detector. In the second step, the number of detection windows, $n$, fused during the clustering process is computed. We compute the confidence of the face detector as in (2).

$$
\mathcal{C}_{d}=\sqrt[n]{\bar{p}}
$$

where $\bar{p}$ is the mean of likelihood values, $p$, computed for all sub-windows that are fused to form a single detection region. It can be observed that as $n$ increases, $\mathcal{C}_{d}$ also increases. Low number of detected sub-windows have been observed for faces that are non-frontal. High values of $\bar{p}$ and $n$ were observed for frontal faces alone.

3. Confidence of the segmentation algorithm $\left(\mathcal{C}_{s g}\right)$. The quantity $\mathcal{C}_{s g}$ is computed as the average of reliability estimate on each landmark of the segmented face. For details on how the reliability of each landmark is computed, readers are referred to [11].

4. Confidence of the classifier $\left(\mathcal{C}_{c}\right)$. If $d_{i}, i \in\{1, \ldots, k\}$ are the distances of the test feature vector to the $k$ nearest training feature vectors $\mathbf{b}_{g}^{i}$, then the confidence that the test image belongs to the class $\mathbb{C}_{j}$ is given by (3).

$$
\mathcal{C}_{c}=\frac{\sum_{\forall i \in \mathbb{D}_{j}} d_{i}^{-1}}{\sum_{\forall i} d_{i}^{-1}}, \quad \mathbb{D}_{j}=\left\{i \mid \mathbf{b}_{g}^{i} \in \mathbb{C}_{j}, i=1, \ldots, k\right\}
$$

All the above confidence values are key measures of the quality of the information obtained from a test image. A low value for any of the above mentioned factors indicates that the information obtained from an image does not accurately represent the current appearance of the user. Realizing this, we adopted the product rule to compute a global score, total confidence, as shown in (4).

$$
\mathcal{C}=\mathcal{C}_{t} \cdot \mathcal{C}_{d} \cdot \mathcal{C}_{s g} \cdot \mathcal{C}_{c}
$$

Further, we define a new confidence measure, system confidence $\left(\mathcal{C}_{s}\right)$, that is an indicator of how confidently the algorithms we use in our FRS make their decisions. The system confidence is defined as in (5).

$$
\mathcal{C}_{s}=\mathcal{C}_{d} \cdot \mathcal{C}_{s g} \cdot \mathcal{C}_{c}
$$

The selection process proceeds as follows. Assume that a test image, with a total confidence $\mathcal{C}$ has been identified as belonging to class $\mathbb{C}_{j}$. It is added to the training set if and only if $\mathcal{C}$ is greater than $\mathcal{C}^{\text {min }}$, namely the minimum total confidence of all the images already enrolled as belonging to class $\mathbb{C}_{j}$. 
Additionally, as images are continuously added to the training set during the update process, it is possible to overwhelm the FRS simply by accumulating a huge amount of training data. In order to limit the number of images per user, every time a new image is added to an already full training set, the image with the lowest total confidence, $\mathcal{C}^{\text {min }}$, is eliminated such that the total number of images per person is utmost $\mathcal{N}$.

\section{$5 \quad$ Experimental Results}

The following experiments aim to test the performance of the FRS when updated using the total confidence, $\mathcal{C}$. Before we present the results, the image database that was acquired to perform the tests is described.

\subsection{Image Acquisition}

The system described in Sec. 3, was used to obtain facial images of 35 individuals (belonging to 15 different nationalities) over a period of five months. A total of 11, 918 images were collected. These images were separated into three categories:

1. Training set. The images in the Training set provide the ground-truth data to enroll users into the system. This set was obtained by selecting 472 images from the 3,498 images captured in the first month. The selection process was performed by a human expert who removed images on which the face detection and segmentation algorithms performed poorly. In other words, the 472 images that were selected for this set had high $\mathcal{C}_{d}$ and $\mathcal{C}_{s}$ values. Each individual in the training set had $11 \pm 5$ images.

2. Update set. This set contains the 6, 420 images captured during the second, third and fourth months. The images from this set, in a chronological order, were used to automatically update the system. No image selection was performed, and therefore, this set may contain images where the detection and segmentation algorithms were inaccurate.

3. Test set. This set contains a total of 2000 images captured during the fifth month. As in the case of the Update set, no selection was performed.

To get an idea of the number of images in which the algorithms we use were inaccurate, we split the images in the Update set and Test set into five categories according to their $\mathcal{C}_{s}$ value. The first category contained images with
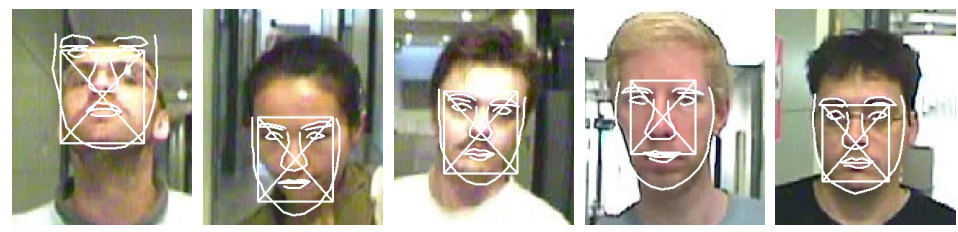

Fig. 3. Example images from the Update set and Test set arranged according to their $\mathcal{C}_{s}$ value. The first, second, third, fourth and fifth images (counted left to right) produced $\mathcal{C}_{s}$ values approximately equal to $0.1,0.3,0.5,0.7$ and 0.9 respectively. 
$\mathcal{C}_{s} \in[0.0 ; 0.2)$, the second contained images with $\mathcal{C}_{s} \in[0.2 ; 0.4)$, and so on. The percentage of images that fell into each category was $5.5 \%, 16.5 \%, 16.5 \%, 18.5 \%$, $43.0 \%$, respectively. Some of the images belonging to each category are shown in Fig. 3. Images with $\mathcal{C}_{s}$ values lower than 0.8 have been observed to cause corruption of the texture data learnt for a user as the texture information obtained is not representative of the user.

\subsection{Training and Update Procedures}

The $k$-NN classifier was trained with the texture parameters obtained from the 472 images in the Training set and their corresponding user IDs. Using the images in the Update set (in chronological order), three types of updates were performed to the Training set:

1. $\mathcal{U C}$. In this case, updates were performed with the total confidence, $C$, as described in Sec. 4.

2. $\mathcal{U C}_{c}$. The update process is similar to that performed in [7. In this scenario, if $\mathcal{C}_{c}$ computed for an image in the Update set is greater than $\mathcal{C}_{c}$ of any of the images in the Training set, then the image will be added to the Training set. Otherwise, the image is ignored.

3. $\mathcal{U C} \mathcal{C}_{t}$. This is a trivial update strategy where newer images from Update set replace older ones in the Training set. In other words, if an image has a $\mathcal{C}_{t}$ value greater than that of any of the images in the Training set, then it is added to the Training set.

During the update procedure, the number of training images belonging to a person is kept fixed to a preset value $\mathcal{N}$ as described in Sec. 4. We repeated our experiments for four different values of $\mathcal{N}: 15,30,60$ and 90 . It was observed for $\mathcal{N}=15$ case that $99.5 \pm 0.78 \%$ of the original images were replaced after the updates. For the cases $\mathcal{N}=30, \mathcal{N}=60$ and $\mathcal{N}=90$, the percentages of replaced images were $91.8 \pm 2.28 \%, 86.3 \pm 2.03 \%$, and $69.3 \pm 1.1 .5 \%$, respectively.

\subsection{Comparison of Accuracy}

The accuracy of the FRS was computed in terms of the correct classification rate, which is a ratio of the number of correctly classified images to the total number of images that are tested. Fig. [4 shows the average scores of classification for the images in the Test set after the updates. Below are four main observations

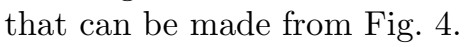

1. Images with high $\mathcal{C}_{s}$ values are classified more accurately than those with low values. This is because, images with high $\mathcal{C}_{s}$ values are more accurately segmented, and therefore, the texture parameters obtained from these images are similar to the ground truth images in the Training set.

2. The accuracy of the FRS after $\mathcal{U C}$ (Fig. 4 a) is higher than the accuracy obtained after $\mathcal{U C}_{c}$ (Fig. 4b) and $\mathcal{U C}_{t}$ (Fig. 45). Since the Test set is the same for all three cases, the decrease in performance after $\mathcal{U C}_{c}$ and $\mathcal{U C}_{t}$ should be a result of a corrupted Training set. Analyzing the Training set 
after $\mathcal{U C}_{c}$ and $\mathcal{U} \mathcal{C}_{t}$, we observed that they contained misclassified images and images with inaccurate segmentation. Such corruption of the training sets never occurred in the training sets corresponding to $\mathcal{U C}$.

3. The standard error measurements (shown as error bars) corresponding to $\mathcal{U C}$ are the smallest (especially when $\mathcal{C}_{s} \geq 0.8$ ). Bigger error bars in Fig. $4 \mathrm{~b}$ result from larger uncertainty in the measurement of correct classification rate. This means that there is higher dispersion in classification rates between the users: some of the users are classified better and some worse. This again is a result of the corruption of training images for some users.

4. In Fig. 4r, the accuracy of the FRS seems to decrease with smaller $\mathcal{N}$. This is because, FRS with low value of $\mathcal{N}$, is more susceptible to corruption. For example, one badly segmented image (belonging to a particular user) in a FRS with $\mathcal{N}=15$ means that $6.6 \%$ of the data belonging to the user is corrupted. However, one badly segmented image in a FRS with $\mathcal{N}=90$ means that only $1.1 \%$ of the data belonging to the user is corrupted. Thus FRS with bigger $\mathcal{N}$ are more robust to corruption. From Fig. 4 (a,b), it can be noticed that the accuracy of the system does not change significantly with $\mathcal{N}$. This indicates that $\mathcal{U C}$ and $\mathcal{U C} \mathcal{C}_{c}$ are better update strategies than $\mathcal{U C} \mathcal{C}_{t}$.

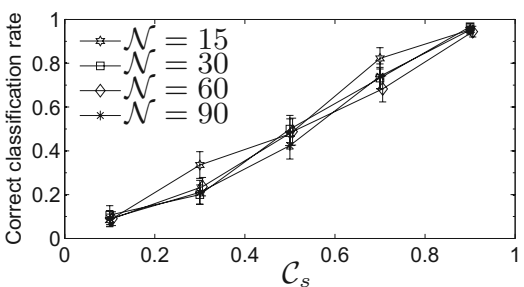

(a)

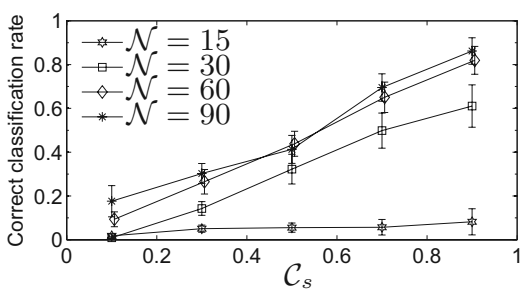

(c)

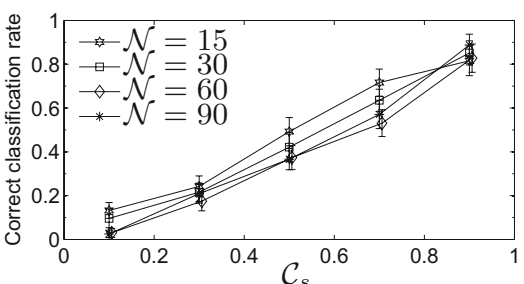

(b)

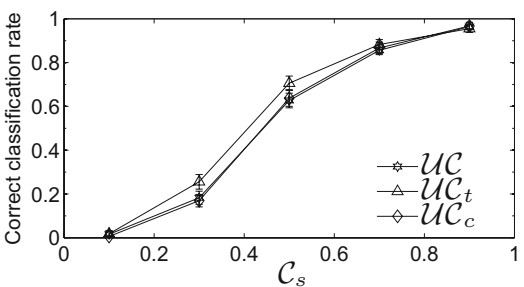

(d)

Fig. 4. Accuracy of the FRS after (a) $\mathcal{U C},\left(\right.$ b) $\mathcal{U C}_{c}$, (c) $\mathcal{U} \mathcal{C}_{t}$. (d) Accuracy of the FRS on the Impostor set with $\mathcal{N}=15$. Error bars represent the standard error of the mean.

Limitations. GEFA does not contain images of users who exhibit abrupt appearance changes. Since images were captured on a day-to-day basis, appearance changes due to to skin tanning and facial hair occur gradually over a sequence of images belonging to a particular user. Abrupt appearance changes generally reduce the accuracy of the FRS. In the present set-up, if the FRS is having 
difficulties in recognizing a legitimate user, then the user is manually enrolled so that the FRS can learn his/her current appearance.

\subsection{Robustness to Impostors}

Robustness of the FRS to impostors was tested on an Impostor set, containing 2,379 images belonging to 74 individuals. These individuals were not part of the Training set, Update set or Test set. No manual selection of images was performed, and therefore, this database contained images where face detection or segmentation algorithms might have been inaccurate.

Robustness to impostors was tested as follows. Every image in the Impostor set was processed by the FRS, and its $\mathcal{C}_{s}$ value was calculated. If its $\mathcal{C}_{s}$ was lower than a preset threshold, then the image was labeled as impostor.

The correct classification rate on the Impostor set is calculated as the ratio of number of images classified as impostors to the total number of images in the Impostor set. Fig. $4 \mathrm{~d}$ plots the correct classification rate of images in the Impostor set vs. the threshold value on $\mathcal{C}_{s}$. It can be seen that if the minimum required $\mathcal{C}_{s}$ value for an image is set to 0.9 , then approximately $95 \%$ of the impostors are classified correctly. Low threshold values on $\mathcal{C}_{s}$ cause misclassifications as the system confuses them with the enrolled users. High threshold values $\left(\mathcal{C}_{s}>0.9\right)$ make FRS restrictive in the sense that even small inaccuracies during detection, segmentation or classification will not be tolerated.

In general, it seems that the system can be run in an optimal point by setting the minimum required $\mathcal{C}_{s}$ value to 0.9 . At this operating point, the system classifies $95 \%$ of the legitimate users (from Fig. 4a) and $95 \%$ of the impostors correctly (from Fig. 4d). This is similar to the performance obtained by many other systems [5] when tested on the XM2VTS database [6].

\section{Conclusions}

We have presented an automatic update rule to adapt the FRS to the continuously varying appearance of faces. Every time the FRS identifies a person, and if it deems that its decision is reliable, the texture paremters obtained from the image are used to maintain the system updated.

The first contribution of this paper is the proposal of the total confidence metric to decide whether an image should be included in the training set or not. The proposed measure intends to quantify how accurately the facial parameters from a test image represent a user. We used the total confidence measure to discard two types of images which are known to cause corruption of training data: images with outdated facial appearance and images where the segmentation algorithm did not extract the facial texture reliably. Finally, this paper presents a new face database, GEFA, in which the appearance of a group of users was captured on a daily basis over five months. This database includes 11, 918 facial images from 35 individuals. It also contains 2,349 images from 74 individuals who act as impostors. Detailed experiments performed on this database show that total confidence is an adequate measure for a system to update itself in an unsupervised manner while avoiding corruption of the training set. 


\section{References}

[1] Aryananda, L.: Recognizing and remembering individuals: online and unsupervised face recognition for humanoid robot. In: IROS 2002, pp. 1202-1207 (2002)

[2] Cootes, T., Edwards, G., Taylor, C.: Active appearance models. IEEE Trans. Pattern Anal. Mach. Intell. 23(6), 681-685 (2001)

[3] Lanitis, A., Taylor, C., Cootes, T.: Modeling the process of ageing in face images. In: ICCV 1999, pp. 131-136 (1999)

[4] Lanitis, A., Taylor, C., Cootes, T.: Toward automatic simulation of aging effects on face images. IEEE Trans. Pattern Anal. Mach. Intell. 24(4), 442-455 (2002)

[5] Messer, K., et al.: Face verification competition on the XM2VTS database. In: Kittler, J., Nixon, M.S. (eds.) AVBPA 2003. LNCS, vol. 2688, pp. 964-974. Springer, Heidelberg (2003)

[6] Messer, K., Matas, J., Kittler, J., Luettin, J., Maitre, G.: XM2VTSDB: The extended M2VTS database. In: AVBPA 1999, pp. 72-77 (1999)

[7] Mou, D., Schweer, R., Rothermel, A.: Automatic databases for unsupervised face recognition. In: CVPRW 2004, pp. 90-97 (2004)

[8] Navarrete, P., Ruiz-del-Solar, J.: Comparative study between different eigenspacebased approaches for face recognition. In: Pal, N.R., Sugeno, M. (eds.) AFSS 2002. LNCS, vol. 2275, pp. 178-184. Springer, Heidelberg (2002)

[9] Perlibakas, V.: Distance measures for PCA-based face recognition. Pattern Recogn. Lett. 25(6), 711-724 (2004)

[10] Phillips, P., et al.: Face recognition vendor test 2002. Evaluation Report (2003), http://www.frvt.org

[11] Sukno, F., Frangi, A.: Exploring reliability for automatic identity verification with statistical shape models. In: AutoID 2007, pp. 80-86 (2007)

[12] Sukno, F., et al.: Active shape models with invariant optimal features. IEEE Trans. Pattern Anal. Mach. Intell. 29(7), 1105-1117 (2007)

[13] Viola, P., Jones, M.: Robust real-time face detection. Int. J. Comput. Vision 57(2), 137-154 (2004) 